\title{
ON THE NEW QUANTIFIERS AND THEIR USE IN MULTICRITERIA DECISION FUNCTIONS VIA ORDERED WEIGHTED AGGREGATIONS
}

\author{
AARTHI CHANDRAMOHAN AND M. V. C. RAO
}

Received 25 August 2005; Accepted 28 November 2005

The goal of this paper is to present new and different quantifiers for ordered weighted aggregation and illustrate their applicability by a real-life example. The role of these operators in the formulation of multicriteria decision making functions, using the concept of quantifier guided aggregation, is also discussed.

Copyright (C) 2006 A. Chandramohan and M. V. C. Rao. This is an open access article distributed under the Creative Commons Attribution License, which permits unrestricted use, distribution, and reproduction in any medium, provided the original work is properly cited.

\section{Introduction}

Yager [7] introduced a new aggregation technique based on the ordered weighted aggregation operators. Let $W$ be a weight vector of dimension $n$, then a mapping OWA ${ }_{w}: R^{n} \rightarrow$ $R$ is an ordered weighted averaging operator (OWA) of dimension $n$ if

$$
\mathrm{OWA}_{w}\left(a_{1}, \ldots, a_{n}\right)=\sum_{i=1}^{n} w_{i} a_{\sigma_{(i)}},
$$

where $\{\sigma(1), \ldots, \sigma(n)\}$ is a permutation of $\{1, \ldots, n\}$ such that $a_{\sigma(i-1)} \geq a_{\sigma(i)}$ for all $a\{2, \ldots$, $n\}$.

Two restrictions are imposed on the OWA operator weights: they must sum to one and they must also lie in the unit interval. In this paper new quantifiers are defined for decision making functions satisfying the above mentioned restrictions.

The OWA operator mentioned in [7] seems to exhibit a number of properties desirable for an aggregation operation:

(1) the OWA operator is symmetric/commutative;

(2) it is mean like. If $F$ is any OWA operator $\operatorname{Min}\left[a_{i}\right] \leq F\left(a_{1}, \ldots, a_{n}\right) \leq \operatorname{Max}\left[a_{i}\right]$. This condition also ensures idem potency, if $a_{i}=a$ for all $i$, then $F\left(a_{1}, \ldots, a_{n}\right)=a$;

(3) it is monotonic; if $a_{i} \geq e_{i}$ for all $i$, then $F\left(a_{1}, \ldots, a_{n}\right) \geq F\left(e_{1}, \ldots, e_{n}\right)$. 
Thus by selecting different weighting vectors, different aggregation imperatives can be implemented. If the weighting vector is $W_{*}$ where $w_{1}=1$ and $w_{n}=0$ for $j \neq 1$, then the aggregated value obtained is $\operatorname{Max}[a]$. If the weighting vector is $W^{*}$ where $w_{n}=1$ and $w_{1}=0$ for $j \neq n$, then the aggregated value obtained is $\operatorname{Min}[a]$. If the weighting vector is $W_{\text {ave }}$ where $w=1 / n$ for all $j$, then the aggregated value is $(1 / n) \sum_{j=1}^{n} a_{j}$.

Yager [7] introduced a characterizing measure $\alpha(W)$ or Orness $(W)$ associated with an OWA operator having a weighting vector $W$, the measure is defined as

$$
\alpha(w)=\frac{1}{n-1} \sum_{i=1}^{n} w_{i}(n-i) .
$$

Orness $\left[w^{*}\right]=1$, Orness $\left[w_{*}\right]=0$, Orness $\left[w_{\text {ave }}\right]=0.5$.

Essentially $\alpha$ can be seen as a measure of "maxness" of the aggregation. A measure of "andness" can be defined as andness $(w)=1-$ Orness $[w]$.

One important use of the OWA operator is an aggregation operator in multicriteria decision making [8]. Central to its use in this domain is the ability of the OWA operator to represent linguistic quantifiers [10] such as most, some, and many. This facility for representing linguistic quantifiers allows to use OWA operators in procedure for constructing decision functions called quantifier guided aggregation. As stated by Zadeh [11] a decision maker is allowed to make a decision in natural language terms such as Most criteria should be satisfied by a good solution.

The remaining of this paper is organized as follows: in Section 2, quantifier guided aggregation is studied and formulated. New quantifiers for OWA operators are briefed in Section 3. The case study: the simulation results are given in Section 4 and finally the discussions and conclusions are drawn in Section 5.

\section{Quantifier guided aggregation}

Bellman and Zadeh [2] introduced fuzzy logic as a tool to develop and model multicriteria decision functions. Zadeh suggested that any relative quantifier can be expressed as a fuzzy subset $Q$ of the unit interval $I$. In this representation for any proportion $y \in I, Q(y)$ indicates the degree to which $y$ satisfies the concept conveyed by the term $Q$. In the theory of generalized quantifiers [1], a generalized quantifier $Q$ is said to be monotonic if a true proposition of the form $p=Q A$ 's is $B$ 's, where $A$ and $B$ are nonfuzzy sets, remains true when $B$ is replaced by any superset (or any subset) of $B$. In this sense, most is a monotonic generalized quantifier under the assumption that $B$ is replaced by a superset of $B$.

A fuzzy quantifier $Q$ is monotone nondecreasing (nonincreasing) if and only if the membership function of $Q, \mu_{Q}$ is monotone nondecreasing over the domain of $Q$. From this definition it follows that

(i) $Q$ is monotone nondecreasing $\Leftrightarrow \geq Q=Q$;

(ii) $Q$ is monotone nonincreasing $\Leftrightarrow \leq Q=Q$,

where $\geq Q$ and $\leq Q$ are treated as "at least $Q$ " and "at most $Q$," respectively.

In this framework the criteria in a problem are represented as fuzzy subsets over the space of decision alternative which enables the use of fuzzy set operators to aggregate the individual criteria to form the overall decision function. When the fuzzy sets representing 
Table 2.1. Properties of various monotonic functions.

\begin{tabular}{lll}
\hline RIM & RDM & RUM \\
\hline$Q(0)=0$ & $Q(0)=1$ & $Q(0)=0$ \\
$Q(1)=1$ & $Q(1)=0$ & $Q(1)=0$ \\
$Q(x) \geq Q(y)$ if $x>y$ & $Q(x) \leq Q(y)$ if $x<y$ & For $a$ and $b \in I, a<b$, \\
& & (i) for $y<a Q(x) \leq Q(y)$ \\
& & if $x<y ;$ \\
& & (ii) for $x \in[a, b], Q(x)=1 ;$ \\
& & (iii) for $x>b, Q(x) \geq Q(y)$ \\
Example: all, & if $x<y$. \\
most, many, at least $\alpha$ & Example: most one, & Example: about $\alpha$ \\
\hline
\end{tabular}

the criteria are combined by an intersection operation, this implicitly implies an "anding" of the criteria and is implementing the imperative that all the criteria must be satisfied by a solution to the problem. As mentioned in [4-6] this strong condition may not always be the appropriate relationship between the criteria. For example, a decision maker may be satisfied if most of the criteria are satisfied; in other cases all they may require is that at least a few of the criteria be satisfied. Yager $[5,6,8]$ suggested a general approach to the formulation of decision functions which he called quantifier guided aggregations. Central to this approach is the concept of linguistic quantifier.

In [9] Yager distinguished three categories of these relative quantifiers used in quantifier guided aggregation, namely, (i) regular increasing monotonic (RIM) quantifier, (ii) regular decreasing monotonic (RDM) quantifier, (iii) regular unimodal (RUM) quantifier. The properties of these three quantifiers are listed in Table 2.1, where the quantifier is represented by a fuzzy subset $Q$.

Assume a decision problem in which there are $n$ collections of criteria of interest. Let these criteria be denoted as $A_{1}, \ldots, A_{n}$. For any possible solution $x$, one can evaluate the degree to which it satisfies the criteria $A_{i}$, which can be denoted as $A_{i}(x) \in[0,1]$. In this framework $A_{i}$ can be viewed as a fuzzy subset over the set of alternatives. In order to determine the appropriateness of a particular alternative $x$ as the solution to the problem, the scores must be aggregated to individual criteria to find some overall single value to associate with the alternative.

In order to obtain this overall evaluation and implement the aggregation, some information must be provided about the relationship between the criteria that are to be aggregated. Yager [6] suggested an approach to this problem which uses

$$
\operatorname{Agg}\left(A_{1}(x), \ldots, A_{n}(x)\right)=\min _{i}\left[A_{i}(x)\right]
$$

Essentially this approach noted above assumes that all the criteria have to be satisfied by an acceptable solution. Once having calculated this value aggregated for all the alternatives the optimal solution is then selected, the alternative with the highest aggregated value. 
Having introduced the OWA operator and the idea of linguistic quantifiers the method of quantifier guided aggregation suggested by Yager [8] can now be described. In the process of quantifier guided aggregation the decision maker provides a linguistic quantifier $Q$ indicating a fuzzy description of the portion of criterion necessary to be satisfied by a good solution. In this approach the decision maker is essentially providing, with the linguistic quantifier, an imperative describing how to aggregate the individual criteria to get an implicit overall decision function that is $Q$ criteria are satisfied by a good solution.

The procedure used to implement this decision imperative is described in the following. The quantifier $Q$ is used to generate an OWA weighting vector of dimension $n$. This weighting vector is then used in an OWA aggregation to determine the overall evaluation for each alternative. For each alternative the argument of this OWA aggregation is the satisfaction of that alternative to each of the $n$ criteria, $A_{i}(x), I=1, \ldots, n$. Algorithmically, the quantifier guided aggregation process is as follows.

Use the quantifier $Q$ to generate a set of weights, $w_{1}, \ldots, w_{n}$.

For each alternative $x$ in the $X$, calculate the overall evaluation

$$
D(x)=F\left(A_{1}(x), \ldots, A_{n}(x)\right)
$$

where $F$ is an OWA aggregation using the weights found in step 1.

The weight generated using linguistic quantifier $Q$ was introduced in [8]; where $Q$ is an RIM quantifier;

$$
W_{i}=Q\left(\frac{i}{n}\right)-Q\left(\frac{i-1}{n}\right) \quad \text { for }(i=1, \ldots, n)
$$

This generates weights which satisfy the two required conditions of OWA weights: $w_{i} \geq 0$ and $\sum_{i=1}^{n} w_{i}=1$. The most commonly used quantifier is $Q(r)=r^{\alpha}, \alpha>0$, a family of RIM quantifiers. In the next section new and different quantifiers are proposed for use in quantifier guided aggregation.

\section{New quantifiers for OWA operators}

In this section new monotonic quantifiers are introduced to be used in weighted aggregation for multicriteria decision making functions. These three new quantifiers satisfy the three basic conditions of OWA aggregation stated below:

$$
\begin{aligned}
& \text { (i) } w_{i} \varepsilon[0,1] \\
& \text { (ii) } \sum_{i=1}^{n} w_{i}=1 \\
& \text { (iii) } \min \left[x_{i}\right] \leq Q(x) \leq \max \left[x_{i}\right]
\end{aligned}
$$

The three new quantifiers are:

(1) quadratic linguistic quantifier (QLQ):

$$
Q_{q}(r)=\left(\frac{1}{1-\alpha(r)^{0.6}}\right)
$$


Table 4.1. Collection of criteria.

\begin{tabular}{l|cc}
\hline Component & Name of the criteria & Criteria \\
\hline \multirow{3}{*}{ Research interests } & Fitness in research group & $C 1$ \\
& On the frontier of research & $C 2$ \\
& Contributions & $C 3$ \\
\hline \multirow{3}{*}{ Academic background } & University & $C 4$ \\
& Grade average & $C 5$ \\
& Duration & $C 6$ \\
\hline
\end{tabular}

(2) exponential linguistic quantifier (ExLQ):

$$
Q_{e}(r)=e^{-r \alpha}
$$

(3) trigonometric linguistic quantifier (TLQ):

$$
Q_{t}(r)=\operatorname{Tan}(r \cdot \alpha) .
$$

The use of quantifiers to guide the aggregation essentially implies that the most criteria better satisfied the solution. Quadratic linguistic quantifier yields better results than any other quantifiers. This condition seems to be the one that is naturally desired in criteria aggregation.

The exponential and trigonometric (ExLQ and TLQ) quantifiers imply that for a good solution it is sufficient if most of the criteria are satisfied. Unlike the existing and quadratic quantifiers, the exponential and trigonometric linguistic quantifier can be seen as an "anding" of the criteria because this quantifier leads to higher weight values towards the end of the weight vector. When such a decision making environment is available these quantifiers can be advantageously employed.

\section{Case study and simulation results}

The applicability of new quantifier guided OWA operators is illustrated by a doctoral student selection problem [3]. The selection process consists of three main components and is briefly summarized below:

(a) the first component is a collection of applicants for the Ph.D. program $X=$ $\{x 1, \ldots, x p\}$

(b) the second component is a collection of six criteria listed in Table 4.1.

All these six criteria are evaluated with three performances: excellent (3), average (2), and week (1). Added to the above six criteria, two more components are also included for selection process:

(i) letters for recommendation Yes No;

(ii) english knowledge Yes No; 
(c) the third component is a group of 11 experts whose opinions are considered for ranking the alternatives.

In the first stage experts provide ranking for each alternative on each criterion so each expert provides a 6-tuple. In the next stage an overall evaluation for an alternative by an expert using OWA is found.

The following rules are used for aggregation purpose:

(i) if an applicant has more than 2 weak performances, then his overall performance should be less than 2;

(ii) if an applicant has maximum two weak performances, then his overall performance should be more than 2;

(iii) if an applicant has all but one excellent performance, then his overall performance should be about 2.75;

(iv) if an applicant has three weak performances and one of them is on the frontier of research, then his overall performance should not be above 1.5.

Rules (iii) and (iv) have priority whenever they are applicable. From first and second rules we get

$$
\begin{aligned}
& F_{\alpha}(3,3,3,1,1,1)=3 \times\left(w_{1}+w_{2}+w_{3}\right)+w_{4}+w_{5}+w_{6}<2, \\
& F_{\alpha}(3,3,3,2,1,1)=3 \times\left(w_{1}+w_{2}+w_{3}\right)+\left(2 \times w_{4}\right)+w_{5}+w_{6}>2 .
\end{aligned}
$$

The value of $\alpha$ and weight vector derived using the existing linguistic quantifier $r^{\alpha}$ and the new quantifiers proposed for the two conditions given in (4.1) are determined as follows.

Case 4.1. For existing linguistic quantifier, $r^{\alpha}$,

$$
w_{i}=\left(\frac{i}{n}\right)^{\alpha}-\left(\frac{i-1}{n}\right)^{\alpha} \text {. }
$$

From (4.1), $\alpha$ is determined as $1<\alpha \leq 1.293$.

Case 4.2. For quadratic linguistic quantifier (QLQ),

$$
\begin{gathered}
Q_{q}(r)=\left(\frac{1}{1-\alpha(r)^{0.6}}\right) \\
w_{i}=\left(\frac{1}{\left(1-\alpha(i / n)^{0.6}\right)}\right)-\left(\frac{1}{\left(1-\alpha((i-1) / n)^{0.6}\right)}\right) .
\end{gathered}
$$

From (4.1), $\alpha$ is determined as $0.481<\alpha \leq 0.50222$.

Case 4.3. For exponential linguistic quantifier (ExLQ),

$$
Q_{e}(r)=e^{-r \alpha}, \quad w_{i}=\left(e^{-\alpha(i / n)}\right)-\left(e^{-\alpha((i-1) / n)}\right) .
$$

From (4.1), we get $-0.7424<\alpha \leq-0.693$. 
Table 4.2. Weight vectors for different quantifiers.

\begin{tabular}{l|ccc|c}
\hline $\begin{array}{l}\text { Weight } \\
\text { vectors }\end{array}$ & $\begin{array}{c}\text { QLQ } \\
\text { (with } \alpha=0.502)\end{array}$ & $\begin{array}{c}\text { ExLQ } \\
\text { (with } \alpha=-0.693)\end{array}$ & $\begin{array}{c}\text { TLQ } \\
\text { (with } \alpha=0.787)\end{array}$ & $\begin{array}{l}\text { Existing quantifier } \\
\text { (with } \alpha=1.2 \text { ) }\end{array}$ \\
\hline$W_{1}$ & 0.205 & 0.1224 & 0.132 & 0.116 \\
$W_{2}$ & 0.144 & 0.1374 & 0.134 & 0.151 \\
$W_{3}$ & 0.144 & 0.1542 & 0.146 & 0.168 \\
$W_{4}$ & 0.154 & 0.1732 & 0.163 & 0.180 \\
$W_{5}$ & 0.168 & 0.194 & 0.1910 & 0.189 \\
$W_{6}$ & 0.19 & 0.2192 & 0.234 & 0.196 \\
$\sum_{i=1}^{6} w_{i}$ & 1.0 & 1.0 & 1.0 & 1.0 \\
\hline
\end{tabular}

Table 4.3. Weight vectors for the best 4 scores.

\begin{tabular}{l|ccc|c}
\hline $\begin{array}{l}\text { Weight } \\
\text { vectors }\end{array}$ & $\begin{array}{c}\text { QLQ } \\
\text { (with } \alpha=0.502)\end{array}$ & $\begin{array}{c}\text { ExLQ } \\
\text { (with } \alpha=-0.693)\end{array}$ & $\begin{array}{c}\text { TLQ } \\
(\text { with } \alpha=0.787)\end{array}$ & $\begin{array}{l}\text { Existing quantifier } \\
\text { (with } \alpha=1.2)\end{array}$ \\
\hline$W_{1}$ & 0.279 & 0.19 & 0.132 & 0.116 \\
$W_{2}$ & 0.213 & 0.213 & 0.134 & 0.151 \\
$W_{3}$ & 0.234 & 0.274 & 0.146 & 0.168 \\
$W_{4}$ & 0.275 & 0.323 & 0.163 & 0.180 \\
$\sum_{i=1}^{6} w_{i}$ & 1.0 & 1.0 & 1.0 & 1.0 \\
\hline
\end{tabular}

Case 4.4. For trigonometric linguistic quantifier (TLQ),

$$
Q_{t}(r)=\operatorname{Tan}(r \cdot \alpha), \quad w_{i}=\left(\operatorname{Tan} \alpha\left(\frac{i}{n}\right)\right)-\left(\operatorname{Tan} \alpha\left(\frac{i-1}{n}\right)\right) .
$$

From (4.1), we get $1.1669<\alpha \leq 1.29457$.

The weight vector results for all the above cases are tabulated in Table 4.2. In the second stage the technique for combining the expert's evaluation to obtain an overall evaluation for each alternative is based upon the OWA operators. Each applicant is represented by an 11-tuple. $\left(b_{1}, \ldots, b_{11}\right)$ where $b_{i} \in[1,3]$. The applicant is evaluated based on his top four scores $\left(b_{1}, \ldots, b_{4}\right)$.

If at least three experts agree that the applicant is excellent, then his final score should be 2.75 which is a cutoff for the best student,

$$
F(3,3,3,2)=3 \times\left(w_{1}+w_{2}+w_{3}\right)+2 \times w_{4}=2.75
$$

If the final score is less than 2, then the applicant is disqualified. The weight vectors for different quantifiers corresponding to (4.6) are listed in Table 4.3. 
Table 4.4. Aggregated scores for proposed and existing quantifiers for a collection of six criteria.

\begin{tabular}{|c|c|c|c|c|c|c|c|c|c|c|}
\hline \multirow{2}{*}{ Experts } & \multicolumn{6}{|c|}{ Performance value for criteria } & \multicolumn{3}{|c|}{ New proposed quantifiers } & \multirow{2}{*}{$\begin{array}{l}\text { Existing } \\
\text { quantifier }\end{array}$} \\
\hline & $\mathrm{C} 1$ & $\mathrm{C} 2$ & C3 & $\mathrm{C} 4$ & C5 & C6 & QLQ & ExLQ & TLQ & \\
\hline Expert 1 & 3 & 2 & 3 & 2 & 3 & 1 & 2.323 & 2.196 & 2.191 & 2.239 \\
\hline Expert 2 & 2 & 3 & 3 & 2 & 3 & 2 & 2.513 & 2.4142 & 2.4254 & 2.435 \\
\hline Expert 3 & 2 & 2 & 3 & 2 & 3 & 2 & 2.034 & 1.9043 & 1.9075 & 1.92 \\
\hline Expert 4 & 3 & 2 & 3 & 3 & 3 & 2 & 2.667 & 2.5874 & 2.5892 & 2.615 \\
\hline Expert 5 & 2 & 2 & 3 & 2 & 3 & 1 & 2.1786 & 2.0417 & 2.0442 & 2.071 \\
\hline Expert 6 & 3 & 2 & 3 & 2 & 3 & 1 & 2.323 & 2.196 & 2.191 & 2.239 \\
\hline Expert 7 & 1 & 2 & 3 & 2 & 3 & 2 & 2.178 & 2.0417 & 2.0442 & 2.071 \\
\hline Expert 8 & 1 & 2 & 3 & 2 & 3 & 1 & 2.009 & 1.8473 & 1.8532 & 1.882 \\
\hline Expert 9 & 1 & 2 & 3 & 2 & 3 & 2 & 2.034 & 1.9043 & 1.9075 & 1.92 \\
\hline Expert 10 & 1 & 2 & 3 & 3 & 3 & 1 & 2.009 & 1.8473 & 1.8532 & 1.882 \\
\hline Expert 11 & 1 & 2 & 3 & 2 & 2 & 1 & 1.658 & 1.5874 & 1.5844 & 1.615 \\
\hline $\begin{array}{l}\text { Final } \\
\text { aggregated } \\
\text { score }\end{array}$ & & & & & & & 2.4815 & 2.3788 & 2.3942 & 2.475 \\
\hline
\end{tabular}

Consider some applicant with the scores given in Table 4.4 for each expert. After reordering in a descending order, the OWA aggregated scores and the final score for the criteria, $C 1, \ldots, C 6$, based on weights are clearly listed in Table 4.4 .

\section{Discussions and conclusion}

Table 4.4 shows the results obtained using new quantifiers and the existing ones for comparison purpose. It is proved that the new quantifiers satisfy the 2 basic conditions imposed on OWA operators. From Tables 4.2, 4.3, and 4.4, it is clear that quadratic linguistic quantifier provides better results than the existing quantifier. The weight values are higher toward the beginning of the weight vector than the existing quantifier. From Table 4.4 it can be seen that the overall aggregated value is higher than all other quantifiers. Hence this quantifier suits best in criteria aggregation. Whereas exponential and trigonometric quantifiers proposed give higher weight values towards the end on the weight vector which implies that it is more like an and operation. Hence from Table 4.4 it is clear that the overall aggregated value is lesser than other quantifiers. But the results are definitely better than the conventional "and" aggregation (all the criteria must be satisfied).

In this paper new linguistic quantifiers for quantifier guided aggregation for use in decision making functions are proposed. Their applicability is clearly illustrated and a comparison is given with the existing one. It has been established that these are as useful as the original one and hence this increases the repertoire of such functions. 


\section{References}

[1] J. Barwise and R. Cooper, Generalized quantifiers and natural language, Linguistics and Philosophy 4 (1981), no. 2, 159-219.

[2] R. E. Bellman and L. A. Zadeh, Decision-making in a fuzzy environment, Management Science 17 (1970/1971), B141-B164.

[3] C. Carlsson, R. Fullér, and S. Fullér, OWA operators for doctoral student selection problem, The Ordered Weighted Averaging Operators: Theory, Methodology, and Applications (R. R. Yager and J. Kacprzyk, eds.), Kluwer Academic, Massachusetts, 1996, pp. 167-178.

[4] J. Kacprzyk and R. R. Yager, Linguistic quantifiers and belief qualification in fuzzy multicriteria and multistage decision making, Control and Cybernetics 13 (1984), no. 3, 155-173.

[5] R. R. Yager, Quantifiers in the formulation of multiple objective decision functions, Information Sciences 31 (1983), no. 2, 107-139.

[6] __ General multiple-objective decision functions and linguistically quantified statements, International Journal of Man-Machine Studies 21 (1984), no. 5, 389-400.

[7] _ On ordered weighted averaging aggregation operators in multicriteria decisionmaking, IEEE Transactions on Systems, Man, and Cybernetics 18 (1988), no. 1, 183-190.

[8] _ Quantifier guided aggregation using OWA operators, International Journal of Intelligent Systems 11 (1996), no. 1, 49-73.

[9] R. R. Yager and D. P. Filev, Essentials of Fuzzy Modeling and Control, John Wiley \& Sons, New York, 1994.

[10] L. A. Zadeh, A computational approach to fuzzy quantifiers in natural languages, Computers \& Mathematics with Applications 9 (1983), no. 1, 149-184.

[11] _ Fuzzy logic = computing with words, IEEE Transactions on Fuzzy Systems 4 (1996), no. $2,103-111$.

Aarthi Chandramohan: Faculty of Information Science and Technology (FIST), Melaka Campus, Multimedia University, 75450 Bukit Beruang, Melaka, Malaysia

E-mail address: aarthi.mohan@mmu.edu.my

M. V. C. Rao: Faculty of Engineering and Technology (FET), Multimedia University, Jalan Ayer Keroh Lama, Bukit Beruang, 75450 Melaka, Malaysia

E-mail address: machavaram.venkata@mmu.edu.my 


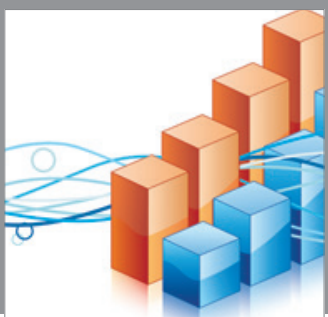

Advances in

Operations Research

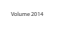

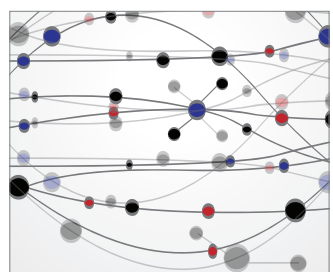

\section{The Scientific} World Journal
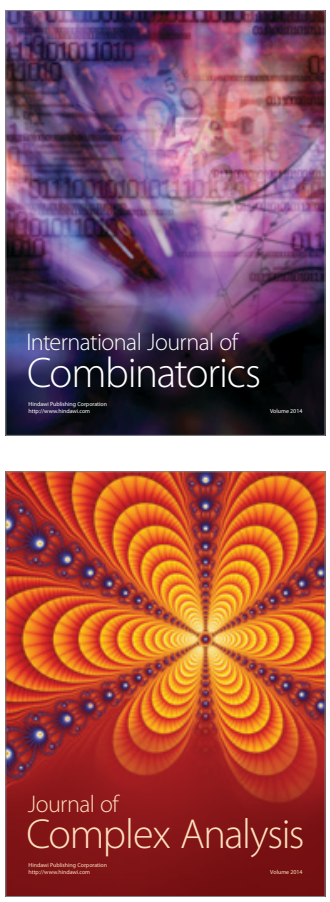

International Journal of

Mathematics and

Mathematical

Sciences
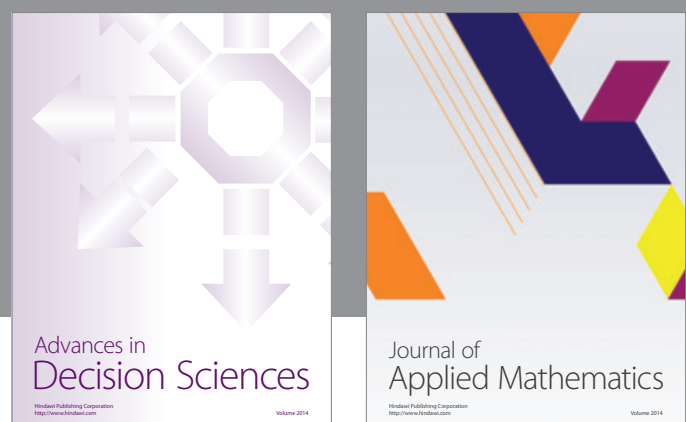

Journal of

Applied Mathematics
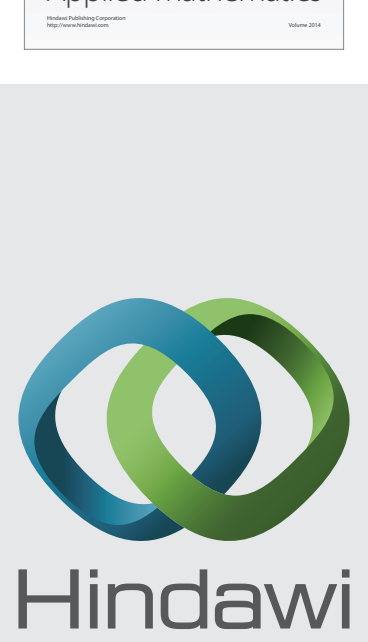

Submit your manuscripts at http://www.hindawi.com
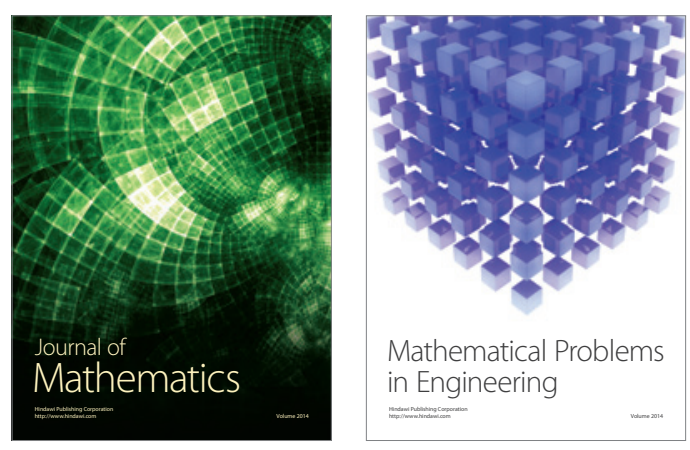

Mathematical Problems in Engineering
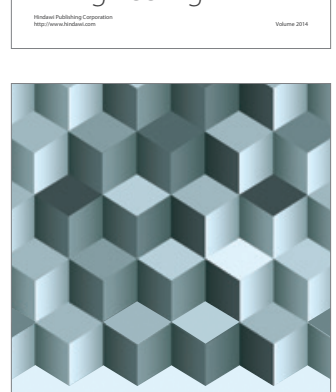

Journal of

Function Spaces
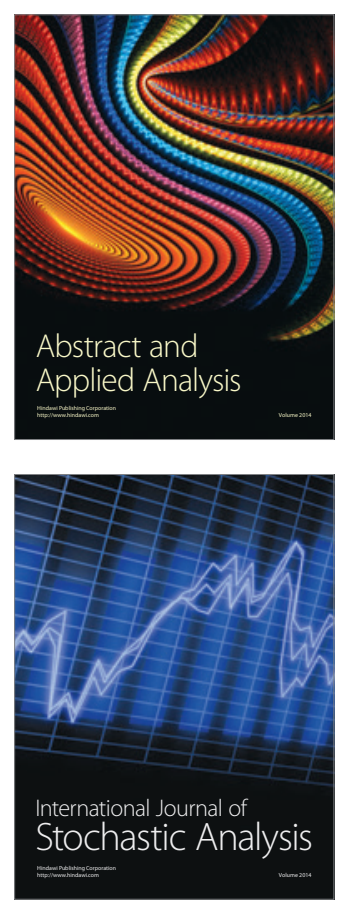

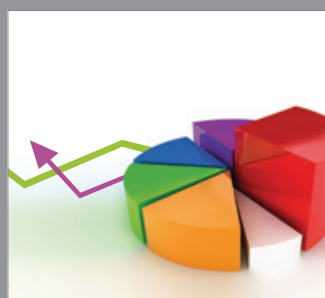

ournal of

Probability and Statistics

Promensencen
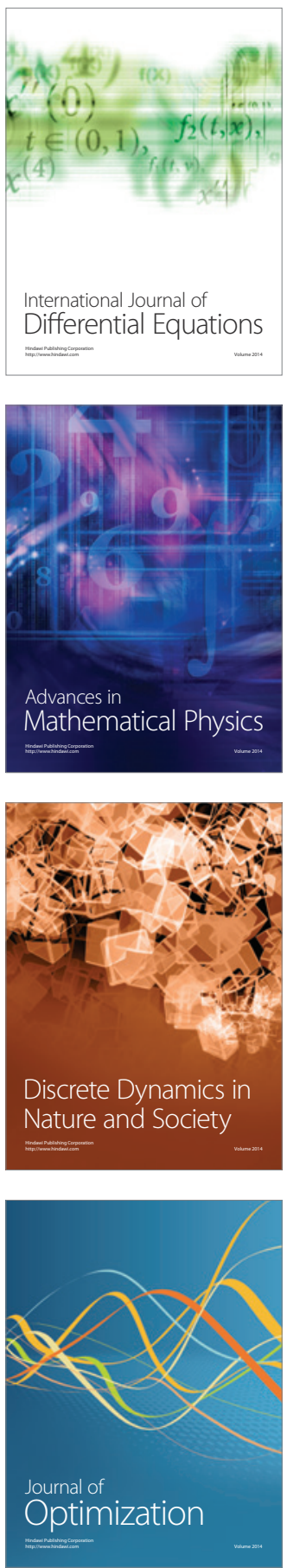